\title{
Anthropometric Variables as Mediators of the Association of Changes in Diet and Physical ActivityWith Inflammatory Profile
}

\begin{abstract}
Gabriela
Cárdenas-Fuentes,

$\mathrm{PhD},{ }^{1}$

Camille

Miguel Ángel Martínez-González, MD, PhD, 2,3,4 María Grau, MD, PhD, 5,6

Lassale,

$\mathrm{PhD},{ }^{1}$ Jordi Salas-Salvadó, MD, PhD, 4,7,8,9, Dolores Corella, MD, PhD, ${ }^{4,10}$ Lluis Serra-Majem, MD, PhD, ${ }^{4,11}$ Julia Warnberg, PhD, ${ }^{4,12}$ Jadwiga Konieczna, PhD, ${ }^{13}$ Ramón Estruch, MD, PhD, ,14 Xavier Pintó, MD, PhD, 4,15 J. Alfredo Martínez, MD, PhD, 4,16,17 Clotilde Vázquez, MD, PhD, ${ }^{4,18}$ Josep Vidal, MD, PhD, ${ }^{19,20}$ Josep A. Tur, MD, PhD, 4,13,21 Andrés Díaz-López, PhD, ,7,9 Hana Lancova, PhD, 22,23 Montserrat Fito, MD, PhD, 1,4,* and Helmut Schröder, PhD 1,6
\end{abstract}

\begin{abstract}
'Cardiovascular Risk and Nutrition Research Group, Hospital del Mar Medical Research Institute (IMIM), Barcelona, Spain. ${ }^{2}$ Department of Preventive Medicine and Public Health, IDISNA, University of Navarra, Pamplona, Spain. ${ }^{3}$ Department of Nutrition, Harvard T.H. Chan School of Public Health, Boston, Massachusetts, USA. ${ }^{4}$ Centro de Investigación Biomédica en Red Fisiopatología de la Obesidad y la Nutrición (CIBEROBN), Institute of Health Carlos III, Madrid, Spain. ${ }^{5}$ Cardiovascular Epidemiology and Genetics Research Group, Hospital del Mar Medical Research Institute (IMIM), Barcelona, Spain. ${ }^{6}$ Centro de Investigación Biomédica en Red Epidemiología y Salud Pública (CIBERESP), Instituto de Salud Carlos III, Madrid, Spain. 'Departament de Bioquímica i Biotecnologia, Human Nutrition Unit, Universitat Rovira i Virgili, Reus, Spain. ${ }^{8}$ Nutrition Unit, University Hospital of Sant Joan de Reus, Spain. ${ }^{9}$ Institut d'Investigació Sanitària Pere Virgili (IISPV), Reus, Spain. ${ }^{10}$ Department of Preventive Medicine, University of Valencia, Spain. ${ }^{1}$ Nutrition Research Group, Research Institute of Biomedical and Health Sciences (IUIBS), University of Las Palmas de Gran Canaria, Spain. ${ }^{2}$ Department of Nursing, School of Health Sciences, University of Málaga-Institute of Biomedical Research in Malaga (IBIMA), Spain. ${ }^{33}$ Health Research Institute of the Balearic Islands (IdISBa), Palma de Mallorca, Spain. ${ }^{14}$ Department of Internal Medicine, Institut d'Investigacions Biomèdiques August Pi Sunyer (IDIBAPS), Hospital Clinic, University of Barcelona, Spain. ${ }^{15}$ Lipids and Vascular Risk Unit, Internal Medicine, Hospital Universitario de Bellvitge, Hospitalet de Llobregat, Barcelona, Spain. ${ }^{16}$ Department of Nutrition, Food Sciences, and Physiology, Center for Nutrition Research, University of Navarra, Pamplona, Spain. ${ }^{17 P r e c i s i o n}$ Nutrition Program on Cardiometabolic Health IMDEA CEI UAM+CSIC, Madrid, Spain. ${ }^{18}$ Department of Endocrinology and Nutrition, Hospital Fundación Jimenez Díaz, Instituto de Investigaciones Biomédicas IISFJD, University Autonoma, Madrid, Spain. ${ }^{19}$ CIBER Diabetes y Enfermedades Metabólicas (CIBERDEM), Instituto de Salud Carlos III (ISCIII), Madrid, Spain. ${ }^{20}$ Department of Endocrinology, Institut d' Investigacions Biomédiques August Pi Sunyer (IDIBAPS), Hospital Clinic, University of Barcelona, Spain. ${ }^{21}$ Research Group on Community Nutrition \& Oxidative Stress, University of Balearic Islands, Palma de Mallorca, Spain. ${ }^{22}$ Servicio Navarro de Salud (Osasunbidea), Pamplona, Spain. ${ }^{23}$ Departament de Salut, CAP Angles de Institut d'Assistència Sanitària, Generalitat de Catalunya, Girona, Spain.
\end{abstract}

*Address correspondence to: Montserrat Fito, MD, PhD, Cardiovascular Risk and Nutrition Research Group, Hospital del Mar Medical Research Institute (IMIM), c/Doctor Aiguader 88, 08003 Barcelona, Spain. E-mail: mfito@imim.es.

Received: July 13, 2020; Editorial Decision Date: February 12, 2021

Decision Editor: Jay Magaziner, PhD, MSHyg

\begin{abstract}
Background: Mechanisms underlying the associations of high levels of physical activity (PA) and adherence to the Mediterranean diet (MedDiet) with a better inflammatory profile remain unclear. Our objective was to assess the mediating role of changes in body mass index
\end{abstract}


(BMI) and waist circumference (WC), as markers of body fat in the association of changes in PA and adherence to the MedDiet, with changes in the inflammatory profile.

Method: This study included 489 adults, aged 55-75 years, from the PREDIMED-Plus multicenter lifestyle intervention trial. An inflammatory score was calculated, based on 8 blood biomarkers: high-sensitivity C-reactive protein, interleukin 6, interleukin 8 , interleukin 18 , monocyte chemo-attractant protein-1, C-peptide, leptin, and regulated on activation, normal T-cell-expressed and secreted chemokine. Biomarkers, levels of PA, score of MedDiet adherence, BMI, and WC were measured at baseline and at 1-year follow-up. Linear regression models were fitted according to the Baron and Kenny framework for mediation analysis.

Results: Changes in BMI and WC mediated the association of both changes in PA and changes in the MedDiet adherence with the inflammatory score. Body mass index mediated $26 \%$ of the association of changes in total PA with the inflammatory profile, and $27 \%$ of the association of changes in the MedDiet, while WC mediated $13 \%$ and $12 \%$ of these associations, respectively.

Conclusion: In older adults at high cardiovascular risk, increasing PA levels and adherence to a MedDiet during 1 year were associated with a lower inflammatory score, which was partly mediated by a reduction in body fat.

Clinical Trials Registration Number: International Standard Randomized Controlled Trial Number: ISRCTN89898870; registration date July 24, 2014, retrospectively registered.

Keywords: Body mass index, Inflammation, Mediation analysis, Mediterranean diet, Waist circumference

Chronic low-grade inflammation has been extensively associated with the pathogenesis of chronic diseases $(1,2)$ and is a hallmark of aging. Therefore, it is imperative to identify the determinants and underlying mechanisms involved in low-grade inflammation. This is particularly relevant in older adults with increased body fat, because aging and obesity are independent risk factors for low-grade inflammation $(3,4)$.

Lifestyle factors have the potential to act on systemic inflammation levels. Evidence indicates that physical activity (PA) interventions are effective at decreasing chronic inflammation in the general population $(5,6)$. In older adults, prospective studies have shown inconsistent results (7-9) and the few available intervention trials have included a relatively small sample size (10-12). The Mediterranean diet (MedDiet) has been associated with lower levels of inflammation (13-18) and lower rates of inflammation-related diseases (1921 ) in both midlife and older adults.

Although higher PA levels and MedDiet adherence are associated with a better inflammatory profile, the mechanisms underlying these associations remain unclear. Evidence indicates that these 2 lifestyle factors are strongly associated with a healthy body mass index (BMI) and waist circumference (WC) (22-24). On the other hand, anthropometric variables reflecting body fat have also shown to be directly associated with low-grade inflammation (25-29). It is therefore plausible to hypothesize that changes in BMI and $\mathrm{WC}$ could mediate the association between changes in PA/MedDiet adherence and changes in low-grade inflammation. However, to the best of our knowledge, only Park et al. have assessed this hypothesis with a cross-sectional study in the general population (30).

The present study aimed to assess, in a 12-month period, the extent to which changes in BMI and in WC mediate the association of changes in PA levels and MedDiet adherence with changes in an array of inflammatory plasma biomarkers in subjects aged 55-75 years. This is particularly relevant in older adults with increased body fat, because aging and obesity are independent risk factors for low-grade inflammation.

\section{Method}

\section{Study Design}

This was a prospective study nested in the ongoing PREDIMEDPlus clinical trial. A detailed description of the study protocol has been published elsewhere $(31,32)$ and further information can be found at http://predimedplus.com/. The effect of the interventions on inflammation in the PREDIMED-Plus pilot study has also been described elsewhere (33). In short, the PREDIMED-Plus is a 6-year, multicenter, randomized controlled trial conducted in Spain assessing the effect of a lifestyle intervention on the primary prevention of cardiovascular diseases (CVDs). Participants were randomly allocated to one of 2 groups: an intensive weight loss intervention program, composed of an energy-restricted MedDiet, PA promotion, and behavioral support, or the control group, receiving traditional health care and a MedDiet recommendation without energy restriction. This clinical trial was registered at the International Standard Randomized Controlled Trial registry (ISRCTN89898870; registration date July 24, 2014).

\section{Study Participants}

From October 2013 to December 2016, 6874 participants were recruited from 23 health centers in Spain. Men aged 55-75 years and women aged 60-75 years at high risk of CVDs were included if they had overweight or obesity (BMI $\geq 27$ and $<40 \mathrm{~kg} / \mathrm{m}^{2}$ ) and met at least 3 components of metabolic syndrome diagnostic criteria, defined according to the International Diabetes Federation and the American Heart Association and National Heart, Lung and Blood Institute (34).

Of the first 1013 participants assessed for eligibility, 143 declined to participate, 36 met an exclusion criterion, and 136 did not meet inclusion or randomization criteria. Of the remaining 698 participants, 70 were excluded due to a protocol change in the prerandomization requirements and 2 were excluded due to a cancer diagnosis. Finally, 134 participants with missing data in the variables included in the present study (at baseline and/or 1-year follow-up) were excluded. Three participants reporting extreme changes in PA (>3 SDs from the mean) were excluded from the final sample. The final sample included 489 participants, with a mean age of $65.5 \pm$ 4.8 years (Supplementary Figure 1).

The Research Ethics Committees of all participating centers approved the study protocol, which was conducted following the standards of the Declaration of Helsinki. All participants provided written informed consent.

\section{Assessment of the Independent Variables}

The Registre Gironí del Cor short-PA questionnaire, validated in Spain (35), was used to measure PA levels. This questionnaire 



Figure 1. Schematic presentation of the mediation models. Waist circumference and body mass index as mediators of the association between changes in total physical activity and changes in the inflammatory score. Adjusted by sex, age, intervention group, smoking status, educational level, trial center, changes in the other lifestyle factor analyzed (MedDiet adherence adjusted by PA and vice versa), and by baseline levels of the corresponding independent and outcome variable. $\mathrm{BMI}=$ body mass index; MedDiet = Mediterranean diet; MET-min/d = metabolic equivalent of task minutes per day; $\mathrm{PA}=$ physical activity; $\mathrm{WC}=$ waist circumference. ${ }^{*} p<.05$; ${ }^{* *} p<.001$; italics $=$ when waist circumference is used as mediator variable.

assesses 4 dimensions of PA: frequency (days per conventional month), duration (minutes per day), intensity (Metabolic Equivalent Task [MET] assigned to each activity), and type (walking at a slow pace [4 METs], gardening [5 METs], brisk walking [5 METs], walking in the countryside [6 METs], climbing stairs [7 METs], and playing sports [11 METs]) (36). Total energy expenditure was quantified as MET minutes per day (MET-min/d), calculated as the sum of the intensity, duration, and frequency assigned to each activity, divided by 30 . The PA was later classified as light ( $<4$ METs), moderate (4-5.5 METs), vigorous ( $\geq 6 \mathrm{METs}$ ), and moderate-to-vigorous PA (MVPA, >4 METs). Adherence to an energy-restricted MedDiet was assessed using the 17-item energy-restricted Mediterranean Diet Adherence Screener (er-MEDAS), as a modified version of the validated 14-item MEDAS (37) which is used to assess adherence to a nonrestricted MedDiet. The modified 17-item screener displays more restrictive cut-offs for caloric-dense items and additional items has been added focusing on reducing caloric intake (32). The list of items and its scoring criteria are presented in Supplementary Table 5. The total score ranges from 0 to 17 , with higher scores indicating a higher adherence. Both exposure variables, PA and MedDiet, were collected by interview by trained dieticians at baseline and at 1 -year follow-up.

\section{Assessment of the Outcome Variable}

At baseline and at 1-year follow-up, trained nurses collected 12-hour overnight fasting blood samples from participants. Samples were kept at $-80^{\circ} \mathrm{C}$ until they were analyzed in a central laboratory. Interleukin
6 (IL-6), IL-8, IL-18, monocyte chemo-attractant protein-1 (MCP1), C-peptide, leptin, and regulated on activation, normal T-cell-expressed and secreted chemokine (RANTES) were simultaneously measured in serum with bead-based multiplexing technology using an XMAG-Luminex assay (Bio-Rad, Hercules, CA) and serum levels of high-sensitivity C-reactive protein (hs-CRP) were measured using a wide-range latex-enhanced immunoturbidimetric assay on an ADVIA 2400 analyzer (Siemens Healthcare Diagnostics Inc., Tarrytown, NY). The lowest detectable values (LDVs) for IL-6, IL-8, IL-18, MCP-1, C-peptide, hs-CRP, leptin, and RANTES were 0.34 $\mathrm{pg} / \mathrm{mL}, 0.36 \mathrm{pg} / \mathrm{mL}, 0.29 \mathrm{pg} / \mathrm{mL}, 0.4 \mathrm{pg} / \mathrm{mL}, 0.09 \mathrm{ng} / \mathrm{mL}, 0.4 \mathrm{mg} / \mathrm{L}$, $0.88 \mathrm{ng} / \mathrm{mL}$, and $0.19 \mathrm{ng} / \mathrm{mL}$, respectively. Values below this LDV were imputed as LDV/2. The number of values imputed at baseline were: 101 values of IL-6, 1 value of C-peptide, 1 value of leptin, and 22 values of hs-CRP; while at follow-up, this values were: 114, 1, 1, and 28 , respectively.

\section{Assessment of Potential Mediators}

Anthropometric variables were measured by trained nurses following the established PREDIMED-Plus protocols. The participants wore light clothing and no shoes. A wall-mounted stadiometer and an electronic scale were used to measured height and weight, respectively. Body mass index was calculated by dividing the weight $(\mathrm{kg})$ by the height squared $\left(\mathrm{m}^{2}\right)$. Waist circumference was measured midway between the lowest rib and the iliac crest after a normal exhalation, using an anthropometric nonelastic tape.

\section{Assessment of Confounders}

Sociodemographic information (age, sex, and educational level) and smoking status were collected at baseline using a general questionnaire. Education was dichotomized as more or less than primary school completion. Smoking was dichotomized as smoker or nonsmoker. Smokers included current smokers or individuals who had stopped smoking less than a year prior to the study baseline.

\section{Statistical Analysis}

Change in the inflammatory score was obtained as follows: (i) quintiles of each biomarker were obtained at baseline and after 1-year follow-up, using the same cut-points (Supplementary Table 1); (ii) the sum of the quintiles of the 8 biomarkers was calculated at baseline and at 1-year follow-up, obtaining a baseline and a follow-up inflammatory score, and (iii) the difference between the baseline and the follow-up inflammatory score was finally calculated. The score ranged from 8 (being in the first quintile for all biomarkers) to 40 (being in the fifth quintile for all biomarkers). Through the inspection of histograms and Q-Q plots, a normal distribution was observed for the baseline inflammatory score, follow-up inflammatory score, and the changes in the inflammatory score.

Linear regression models were fitted to analyze the associations of changes in PA (total PA, light PA, and MVPA) and in MedDiet adherence with changes in the inflammatory score. Models included sex, age, intervention group, smoking status, educational level, trial center, changes in the other lifestyle factor (models using changes in MedDiet score as the independent variable were adjusted for changes in total PA and vice versa), baseline levels of the corresponding independent and outcome variables, and, when appropriate, by changes in the intensities of PA. Including interaction terms in the linear regression models allowed the assessment of the influence of sex, age ( $\leq 65$ and $>65$ years old), educational level (more or less than primary school completion), and study group (weight loss intervention or 
control) in the association between changes in the 2 lifestyle factors analyzed and changes in the inflammatory score. To detect if BMI and WC were potential mediators, we further adjusted the models for either BMI or WC. We primarily presented unstandardized coefficients, but also presented standardized coefficients as Supplemental Material to compare the magnitude of the associations of PA and MedDiet. For this purpose, we standardized the continuous variables (changes in total PA, MVPA, light PA, and adherence to the MedDiet) and ran the models using the resulting $z$ scores.

Mediation analysis was performed to study the extent to which anthropometric variables (BMI and WC) were responsible for the association between changes in lifestyle factors (PA and MedDiet) and changes in the inflammatory profile. This analysis was based on the standardized steps proposed by Baron and Kenny (38) (Figures 1 and 2). The following models were fitted: (i) a linear regression assessing the association between changes in PA and in the inflammatory score, excluding the mediators (changes in $\mathrm{BMI}$ or in $\mathrm{WC}$ ) from the model to check that there is an effect that may be mediated (path c); (ii) a linear regression assessing the association between changes in PA and in the anthropometric markers (BMI or WC), excluding the inflammatory score from the model (path a); and (iii) a linear regression assessing the association between change in the anthropometric markers (BMI or WC) and in the inflammatory score, with additional adjustment for changes in PA (path b). The same procedure was repeated using changes in light PA, MVPA, and MedDiet adherence as the independent variables. To assess the


Figure 2. Schematic presentation of the mediation models. Waist circumference and body mass index as mediators of the association between changes in MedDiet adherence and changes in the inflammatory score. Adjusted by sex, age, intervention group, smoking status, educational level, trial center, changes in the other lifestyle factor analyzed (MedDiet adherence adjusted by PA and vice versa), and by baseline levels of the corresponding independent and outcome variable. BMI = body mass index; MedDiet $=$ Mediterranean diet; MET-min/d = metabolic equivalent of task minutes per day; $\mathrm{PA}=$ physical activity; $\mathrm{WC}=$ waist circumference. ${ }^{*} p<.05$; ${ }^{* *} p<.001$; italics $=$ when waist circumference is used as mediator variable. presence of collinearity between the independent variables included in the models, the variance inflation factor was calculated.

The existence of mediation was determined by analyzing the direct effect, which represents the association between the independent and dependent variable while the mediator is held constant (path $c^{\prime}$ ), and the indirect effect (Path $\mathrm{a} \times$ Path $\mathrm{b}$ ) which represents the amount of mediation exerted by the mediator variable (BMI or WC) in the association between the independent and dependent variable.

We further estimated the proportion mediated by BMI and WC, dividing the indirect effect by the sum of the direct and indirect effect. To calculate the significance of these estimations, confidence intervals (CIs) were obtained from bootstrapping analysis (1000 replications). Since the aim of this study was not to analyze the role of the intervention in the studied mediation, specific methods to address potential bias related to mediation analysis in randomized clinical trials $(39,40)$ were not required. Nevertheless, we tested the potential influence of the intervention arm by including an interaction term in one set of models, and by performing a sensitivity analysis removing the intervention variable from the models. Additionally, we performed sensitive analysis to test for bias due to missing data.

Associations were considered significant if $p<.05$. Mediation was assessed by the R package "mediation" version 4.4.6. All the statistical analysis was performed with $\mathrm{R}$, version 3.0.2.

\section{Results}

After 1 year of follow-up, participants reported a mean $(S D)$ change in total PA of +67 (372) MET-min/d (+87 [427] MET-min/d in men and +49 [314] MET-min/d in women). The mean change in light PA and MVPA was +6 (159) MET-min/d and +61 (362) MET-min/d, respectively, while the mean change in MedDiet adherence was 2.5 (3.1) points. Baseline characteristics of the participants are outlined in Table 1. The median (interquartile ranges) of the baseline levels of total PA, light PA, and MVPA were 316 (130-539), 64 (0-160), and $195(40-412)$ METs-min/d, while the mean $(S D)$ for the baseline levels of MedDiet score was 8.9 (2.5). At follow-up, those values were 375 (212-627), 80 (0-200), 247 (44-503) for total PA, light PA, and MVPA, respectively, and 11.5 (2.8) for the MedDiet.

Changes in total PA were inversely associated with changes in the inflammatory profile, adjusted for sex, age, intervention group, smoking status, educational level, study center, changes in MedDiet adherence, baseline PA levels, and baseline inflammatory score (path c) (Table 2). When additionally adjusting by BMI or WC, the magnitude of the association decreased, but remained significant. Similar findings were observed when changes in total PA were replaced by changes in MVPA and in MedDiet adherence. To analyze the interaction between the exposure and sex, an interaction term was added in each of the 3 models presented in Table 2. Therefore, a total of 6 models were fitted: 3 models with changes in total PA and 3 models with changes in MedDiet as exposure variable. The same procedure was followed for the test of interaction with age, education, and intervention group. This analysis showed that the association between changes in the studied lifestyle factors and in the inflammatory score was not influenced by sex, age, education, or intervention group ( $p$ value of all interaction terms $>.05$, data not shown). The analysis including standardized predictors showed that changes in total, MVPA, and MedDiet adherence were associated with changes in the inflammatory profile to a similar extent (Supplementary Table 2).

A summary of the mediation analysis is depicted in Figure 1. There was a significant association between changes in total PA and 
Table 1. Baseline Characteristics of the Study Participants $(n=489)^{\mathrm{a}}$

\begin{tabular}{lc}
\hline Characteristic & \\
\hline Women, $n$ & $259(53.0 \%)$ \\
Age, $y$ & $65.5(65.0-65.9)$ \\
Study group, $n$ & \\
$\quad$ Intervention & $256(52.4 \%)$ \\
Control & $233(47.6 \%)$ \\
More than primary education, $n$ & $237(48.5 \%)$ \\
BMI, kg/m ${ }^{2 b}$ & $32.4(32.1-32.7)$ \\
WC, cm & $106.8(106.0-107.7)$ \\
Smokers, $\mathrm{n}^{\mathrm{c}}$ & $68(13.9 \%)$ \\
MedDiet score & \\
PA, MET-min/d & $8.9(8.7-9.1)$ \\
Total & $316(130-539)$ \\
Light & $64(0-160)$ \\
Moderate to vigorous & $195(40-412)$ \\
Markers of inflammation & \\
IL-6 pg/mL & $1.33(0.55-2.15)$ \\
IL-8 pg/mL & $8.07(5.91-10.63)$ \\
IL-18 pg/mL & $80.26(58.10-107.49)$ \\
MCP-1 pg/mL & $67.18(47.44-88.37)$ \\
C- peptide ng/mL & $1.55(1.50-1.60)$ \\
Hs-CRP mg/L & $2.37(1.28-4.95)$ \\
Leptin ng/mL & $14.80(8.21-26.34)$ \\
RANTES ng/mL & $10.13(9.99-10.28)$ \\
\hline
\end{tabular}

Notes: $\mathrm{BMI}=$ body mass index; hs-CRP $=$ high-sensitivity C-reactive protein; $\mathrm{IL}=$ interleukin; $\mathrm{MCP}-1$ = monocyte chemo-attractant protein-1; MedDiet score $=$ adherence to an energy-restricted Mediterranean diet; MET$\mathrm{min} / \mathrm{d}=$ metabolic equivalent of task minutes per day; $\mathrm{PA}=$ physical activity; RANTES = regulated on activation, normal T-cell-expressed and secreted chemokine; $\mathrm{WC}=$ waist circumference.

${ }^{a}$ Categorical, continuous normal, and continuous nonnormal distributed variables are expressed as number (proportion), mean (confidence interval), and median (interquartile range), respectively.

${ }^{\mathrm{b}} \mathrm{BMI}$ was calculated by dividing the weight (kilograms) by the square of the height (meters).

'Smokers included current smokers and ex-smokers who stopped smoking less than a year before baseline.

${ }^{\mathrm{d}}$ MedDiet score ranges from 0 (minimum adherence) to 17 (maximum adherence).

changes in BMI and WC ( $\beta$ coefficient: -0.08 and -0.26 respectively, path a) and between changes in these anthropometric markers and in the inflammatory score ( $\beta$ coefficient: 0.63 and 0.10 , respectively, path b). Regarding PA intensity, changes in MVPA were inversely associated with changes in BMI and in WC, whereas changes in light PA were only associated with changes in WC (Supplementary Table 3). Similar significant associations were found when changes in MedDiet adherence were used as the independent variable. The variance inflation factor (VIF) of each of the variables included in Models a, b, and c was below 2 (data not shown).

After observing the statistically significant associations in paths $\mathrm{a}, \mathrm{b}$, and $\mathrm{c}$-with the exception of the nonsignificant association between changes in light PA and changes in $\mathrm{BMI}$ - we proceeded to fit the mediation model. Changes in BMI significantly mediated the association between changes in total PA and those in the inflammatory score, explaining $26 \%$ (CI: $5 \%-85 \%$ ) of the overall association (Table 3). Additionally, a direct effect was observed between changes in total PA and those in the inflammatory score ( $\beta$ coefficient $[95 \% \mathrm{CI}]=-0.13[-0.25$ to -0.01$])$. Changes in BMI mediated the association of changes in MVPA and in MedDiet adherence with changes in the inflammatory score (24\% [CI: $4 \%-94 \%$ ] and $27 \%$
[CI: $13 \%-100 \%$ ], respectively]. Changes in WC also mediated the association of changes in total PA, MVPA, and MedDiet adherence with changes in the inflammatory profile, accounting for $13 \%$ (CI: $4 \%-44 \%$ ), $11 \%$ (CI: $3 \%-43 \%$ ), and $12 \%$ (CI: $3 \%-36 \%$ ) of the association, respectively. When removing the intervention arm from the mediation model, we observed a moderate increase in the proportion mediated by BMI and a moderate decrease in those mediated by WC. For example, in the association between changes total PA and changes in the inflammatory score, the proportion mediated by changes in BMI increased from $26 \%$ to $32 \%$ and in the association between changes in the MedDiet and in the inflammatory score the proportion mediated by changes in BMI, decreased from $27 \%$ to $12 \%$ (Supplementary Table 4).

\section{Discussion}

In this prospective study, we assessed the role of markers of global and abdominal adiposity as potential mediators of the association between changes in 2 lifestyle factors and in the inflammatory profile in older adults with metabolic syndrome. Body mass index acted as a partial mediator of the association of changes in total PA and in MedDiet adherence with changes in the inflammatory profile (the proportion mediated was $26 \%$ and $27 \%$, respectively). To a lesser extent, WC also acted as a partial mediator of these associations (13\% and $12 \%$, respectively).

Despite the known associations of diet and PA with inflammation, and of adiposity with both lifestyle and inflammation, the mediating role of anthropometric variables in the association between lifestyle and inflammation has been little studied. Most studies have reported associations of either PA or dietary intake with inflammatory markers independently of anthropometric factors but did not evaluate mediation per se $(5,27,41)$.

One study by Fedewa et al. (5) analyzed anthropometric markers as moderators of the causal effect between PA interventions and CRP levels in a meta-analysis of 83 intervention trials. They found that when PA interventions resulted in a decreased BMI, the magnitude of CRP reduction was greater than when PA interventions did not result in decreased BMI; nonetheless, the CRP decrease was significant in both BMI groups. Similarly, Richard et al. (18) reported that a high MedDiet adherence effectively reduced inflammation, and this effect was amplified when WC decreased. These findings could indicate that changes in BMI and in WC explain partly the association between PA/MedDiet adherence and inflammation. In the same line, the present study showed that the association between changes in PA/MedDiet adherence and in inflammation was reduced after adjusting for changes in BMI or in WC, supporting the hypothesis that changes in BMI and/or WC could be a mechanism by which $\mathrm{PA} / \mathrm{MedDiet}$ adherence is related to inflammation.

To the best of our knowledge, only Park et al. (30) have performed a mediation analysis, although they used an observational cross-sectional design. In 4700 individuals aged 20-90 years, they assessed BMI and WC mediation of a cross-sectional association between MedDiet adherence and inflammatory markers. They found that WC mediated the association of MedDiet adherence with white blood cell count and fibrinogen $(16.9 \%$ and $9.6 \%$, respectively), while BMI mediated a nonsignificant proportion of these associations $(13.1 \%$ and $7.6 \%$, respectively). On the contrary, in the present study, we found a greater mediation effect of BMI than WC. This finding was not expected because the accumulation of visceral fat has shown a greater correlation with 
Table 2. Association Between Changes in Lifestyle Factors (Adherence to the MedDiet and Total, Light, and Moderate-to-Vigorous PA) and Changes in the Inflammatory Score

\begin{tabular}{|c|c|c|c|}
\hline & \multicolumn{3}{|l|}{$\Delta$ Inflammatory Score ${ }^{\mathrm{b}}$} \\
\hline & Model 1 & Model 2 & Model 3 \\
\hline & $\beta$ Coefficient $(95 \% \mathrm{CI})$ & $\beta$ Coefficient $(95 \%$ CI $)$ & $\beta$ Coefficient $(95 \% \mathrm{CI})$ \\
\hline \multicolumn{4}{|l|}{$\Delta \mathrm{PA}(100 \mathrm{MET}-\mathrm{min} / \mathrm{d})$} \\
\hline Total & $-0.19(-0.30 \text { to }-0.07)^{*}$ & $-0.14(-0.26 \text { to }-0.03)^{*}$ & $-0.17(-0.29 \text { to }-0.06)^{*}$ \\
\hline Light & $0.005(-0.29$ to 0.30$)$ & $0.06(-0.23$ to 0.35$)$ & $0.04(-0.25$ to 0.33$)$ \\
\hline Moderate to vigorous & $-0.19(-0.31 \text { to }-0.08)^{*}$ & $-0.15(-0.27 \text { to }-0.04)^{*}$ & $-0.18(-0.29 \text { to }-0.06)^{*}$ \\
\hline$\Delta$ MedDiet score ${ }^{c}$ & $-0.25(-0.41 \text { to }-0.10)^{*}$ & $-0.18(-0.34 \text { to }-0.04)^{*}$ & $-0.23(-0.39 \text { to }-0.08)^{*}$ \\
\hline
\end{tabular}

Notes: $95 \%$ CI = 95\% confidence interval; MedDiet = Mediterranean diet; MET-min/d = metabolic equivalent of task minutes per day; PA = physical activity.

aLinear regression models were designed as follows: Model 1 was adjusted by sex, age, intervention group, smoking status, educational level, trial center, changes in the other lifestyle factor (MedDiet adherence adjusted by PA and vice versa), baseline levels of the corresponding independent variable (adherence to the MedDiet and total, light, and moderate-to-vigorous PA), baseline level of the inflammatory score, and, when appropriate, by changes in the different intensities of PA. Model 2 was adjusted for all covariates in Model 1 and for changes in body mass index. Model 3 was adjusted by all covariates in Model 1 and for changes in waist circumference. Values indicate the $\beta$ coefficient $(95 \% \mathrm{CI})$ of the change in the inflammatory score, occurring with each increase in $100 \mathrm{MET}$-min/d of PA or 1 point in the adherence to the MedDiet. After including interaction terms of sex, age, intervention group, and education with the independent variable (ie, Sex $\times$ Changes in PA) separately, no significant interaction effect was found.

${ }^{\text {b}}$ The inflammatory score ranged from 8 (minimum inflammatory state) to 40 (maximum inflammatory state).

${ }^{c}$ MedDiet score ranged from 0 (minimum adherence) to 17 (maximum adherence).

" $p<.05$.

inflammation, compared to the accumulation of general body fat (42). One possible explanation is the greater inaccuracy in WC measurements in comparison with height and weight alone, particularly in subjects with abdominal obesity (43). Moreover, despite commonly thought to be a good marker of visceral fat, WC is also a marker of abdominal subcutaneous fat (44). This is supported by Mayr et al. (22), who found that a MedDiet intervention was associated with a decrease in WC but not in visceral fat. This is important because the changes in WC observed in our study were not necessarily related to visceral adipose tissue, which is more closely associated with inflammation than is general adipose tissue.

The nonsignificant association between changes in light PA and changes in the inflammatory score could be explained by the minor changes reported in light PA during the study period (mean change +6 [159] MET-min/d). On the other hand, previous studies $(45,46)$ have also shown that light PA is not consistently associated with inflammation; therefore, the recommendation of MVPA when aiming to reduce the levels of inflammation seems more appropriate.

Adipose tissue has endocrine functions and is an active secretor of proinflammatory and chemotactic compounds (47). Our findings add further evidence to the notion that changes in surrogate markers of body fat play a crucial role in the mechanism involved in the association between changes in lifestyle factors and the inflammatory profile.

Nevertheless, it should be noted that BMI and WC mediated less than $30 \%$ of the associations studied. Several mechanisms other than changes in anthropometric variables could explain the association of changes in PA and MedDiet adherence with concurrent changes in the inflammatory profile. According to Bailey and Holscher (48), changes in the gastrointestinal microbiota and a decrease in the circulating levels of bacterial endotoxins could be the 2 additional mechanisms explaining the association between a high adherence to the MedDiet and inflammation. On the other hand, Casas et al. $(16,17)$ suggested that the synergistic effect of key foods in the MedDiet, including nuts and extra virgin olive oil, could explain this anti-inflammatory effect. Mechanisms that could explain the association between PA and inflammation include a PA-related antioxidant and antiatherogenic effect (49), an improved endothelial function, and enhanced insulin sensitivity (50). Finally, it has been suggested that high levels of PA and MedDiet adherence are associated with a reduction in the expression of proinflammatory and proatherogenic genes, including epigenetic changes that are among the mechanisms responsible for the decrease in inflammation $(51,52)$.

The use of 8 biomarkers and the prospective nature of the analysis are strengths of the study. The main limitations in this study were (i) the potential multiplicity derived by the simultaneous testing of multiple mediation hypothesis, (ii) the potential bias induced by the indirect nature of the measurements of abdominal obesity, and (iii) the alternative analysis, other than mediation, that could address the complex association between lifestyle behaviors, anthropometric markers, and inflammation that were not tested in this study. Finally, although using self-reported questionnaires to measure PA and adherence to the MedDiet can lead to errors and misclassification, the errors were most likely random, which would underestimate our results.

In conclusion, this study showed that changes in PA and in MedDiet adherence, associated with changes in the inflammatory score, were partly mediated by changes in anthropometric measures. The monitoring of surrogate markers of body fat, in addition to the promotion of PA and MedDiet adherence, could be an effective strategy to control the inflammatory profile.

\section{Supplementary Material}

Supplementary data are available at The Journals of Gerontology, Series A: Biological Sciences and Medical Sciences online.

\section{Funding}

This work was supported by the Spanish Ministry of Health (Carlos III Health Institute) through the Fondo de Investigación para la Salud (FIS), which is cofunded by the European Regional Development Fund (2 coordinated FIS projects [led by J.S.-S. and J.V.] funded by the following grant codes: 
Table 3. Mediation Analysis for the Association Between Changes in Lifestyle Factors (MedDiet Adherence andTotal, Light, and Moderateto-Vigorous PA) and Changes in the Inflammatory Score, Through Anthropometric Measures ${ }^{\mathrm{a}}$

\begin{tabular}{|c|c|c|c|c|c|}
\hline \multirow[t]{2}{*}{ Independent Variable } & \multirow[t]{2}{*}{ Mediator } & \multirow[t]{2}{*}{ Outcome Variable } & \multirow{2}{*}{$\frac{\text { Indirect Effect }}{\beta \text { Coefficient }(95 \% \mathrm{CI})}$} & \multirow{2}{*}{$\frac{\text { Direct Effect }}{\beta \text { Coefficient }(95 \% \mathrm{CI})}$} & \multirow{2}{*}{$\frac{\text { Proportion Mediated }}{\%}$} \\
\hline & & & & & \\
\hline \multicolumn{6}{|l|}{$\Delta \mathrm{PA}(100 \mathrm{MET}-\mathrm{min} / \mathrm{d})$} \\
\hline Total & $\Delta \mathrm{BMI}$ & $\begin{array}{l}\Delta \text { Inflammatory } \\
\text { score }\end{array}$ & $-0.05(-0.08 \text { to }-0.01)^{*}$ & $-0.13(-0.25 \text { to }-0.01)^{*}$ & $25.8 \%(5.4-85.4 \%)^{*}$ \\
\hline Light & $\Delta \mathrm{BMI}$ & $\begin{array}{l}\Delta \text { Inflammatory } \\
\text { score }\end{array}$ & $-0.06(-0.12$ to 0.00$)$ & $0.07(-0.20$ to 0.34$)$ & $\begin{array}{l}11.8 \%(-100.0 \text { to } \\
100.0 \%)\end{array}$ \\
\hline Moderate to vigorous & $\Delta \mathrm{BMI}$ & $\begin{array}{l}\Delta \text { Inflammatory } \\
\text { score }\end{array}$ & $-0.05(-0.09 \text { to }-0.01)^{*}$ & $-0.14(-0.26$ to 0.00$)$ & $23.7 \%(4.2-93.7 \%)^{*}$ \\
\hline$\Delta$ MedDiet score ${ }^{c}$ & $\Delta \mathrm{BMI}$ & $\begin{array}{l}\Delta \text { Inflammatory } \\
\text { score }\end{array}$ & $-0.07(-0.12 \text { to }-0.03)^{*}$ & $-0.18(-0.32$ to 0.00$)$ & $26.7 \%(13.3-100.0 \%)^{*}$ \\
\hline \multicolumn{6}{|l|}{$\Delta \mathrm{PA}(100 \mathrm{MET}-\mathrm{min} / \mathrm{d})$} \\
\hline Total & $\Delta \mathrm{WC}$ & $\begin{array}{l}\Delta \text { Inflammatory } \\
\text { score }\end{array}$ & $-0.03(-0.05 \text { to }-0.01)^{*}$ & $-0.16(-0.27 \text { to }-0.05)^{* *}$ & $12.8 \%(4.0-43.8 \%)^{*}$ \\
\hline Light & $\Delta \mathrm{WC}$ & $\begin{array}{l}\Delta \text { Inflammatory } \\
\text { score }\end{array}$ & $-0.05(-0.10 \text { to } 0.00)^{*}$ & $0.05(-0.22$ to 0.30$)$ & $\begin{array}{l}13.9 \%(-100.0 \text { to } \\
10.00 \%)\end{array}$ \\
\hline Moderate to vigorous & $\Delta \mathrm{WC}$ & $\begin{array}{l}\Delta \text { Inflammatory } \\
\text { score }\end{array}$ & $-0.02(-0.05 \text { to }-0.01)^{*}$ & $-0.17(-0.29 \text { to }-0.04)^{*}$ & $11.1 \%(2.8-42.6 \%)^{*}$ \\
\hline$\Delta$ MedDiet score ${ }^{c}$ & $\Delta \mathrm{WC}$ & $\begin{array}{l}\Delta \text { Inflammatory } \\
\text { score }\end{array}$ & $-0.03(-0.06 \text { to }-0.01)^{*}$ & $-0.22(-0.36 \text { to }-0.04)^{*}$ & $11.8 \%(3.4-36.2 \%)^{*}$ \\
\hline
\end{tabular}

Notes: $95 \%$ CI = 95\% confidence interval; BMI = body mass index; MedDiet = Mediterranean diet; MET-min/d = metabolic equivalent of task minutes per day; $\mathrm{PA}=$ physical activity; $\mathrm{WC}=$ waist circumference.

aThe following models were fitted for mediation analysis: (i) a linear regression with changes in total PA as the independent variable and changes in the inflammatory score as the outcome variable, excluding the mediator from the model (path c); (ii) a linear regression with changes in total physical activity as the independent variable and changes in the anthropometric marker (BMI or WC) as outcome variable, excluding the inflammatory score from the model (path a); and (iii) a linear regression with changes in the anthropometric marker (BMI or WC) as independent variable and changes in the inflammatory score as outcome variable, adjusted by changes in total PA (path b). The same procedure was repeated using light PA, moderate-to-vigorous PA, and adherence to a MedDiet as independent variables. All 3 models were further adjusted by sex, age, intervention group, smoking status, educational level, trial center, changes in the other lifestyle factor when one of them was used as the independent variable (MedDiet adherence by PA and vice versa), baseline levels of the corresponding independent and outcome variables, and, when appropriate, by changes in the intensities of PA. The direct effect represents the association between the independent and dependent variable while the mediator is held constant (path $\mathrm{c}^{\prime}$ ), and the indirect effect is calculated as Path $\mathrm{a} \times$ Path $\mathrm{b}$. Values indicate the $\beta$ coefficient $(95 \% \mathrm{CI}$ ) of the change in the inflammatory score, occurring with each increase in $100 \mathrm{MET}$-min/d of PA or 1 point in the adherence to the MedDiet.

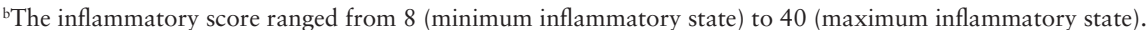

'MedDiet score ranged from 0 (minimum adherence) to 17 (maximum adherence).

$" p<.05$.

PI13/00673, PI13/00492, PI13/00272, PI13/01123, PI13/00462, PI13/00233, PI13/02184, PI13/00728 PI13/01090 PI13/01056, PI14/01722, PI14/00636, PI14/00618, PI14-00696, PI14/01206, PI14/01919, and PI14/00853); the European Research Council (Advanced Research Grant 2013-2018, grant number 340918); Recercaixa (2013ACUP00194); Consejería de Salud de la Junta de Andalucía (PI0458/2013); and a SEMERGEN grant. None of the funding sources took part in the design, collection, analysis or interpretation of the data, or in the decision to submit the manuscript for publication. CIBERobn (Centros de Investigación Biomédica en Red: Obesidad y Nutrición), CIBEResp (Centros de Investigación Biomédica en Red: Epidemiologia y Salud Publica), and CIBERdem (Centros de Investigación Biomédica en Red: Diabetes y Enfermedades Metabólicas asociadas) are initiatives of Carlos III Health Institute, Madrid, Spain.

\section{Conflict of Interest}

Dr. Salas-Salvadó reports serving on the board of and receiving grant support through his institution from the International Nut and Dried Fruit Council, and Eroski Foundation. Reports serving in the Executive Committee of the Instituto Danone Spain and on the Scientific Committee of the Danone International Institute. He has received research support from Patrimonio Comunal Olivarero, Spain; and Borges S.A., Spain. Reports receiving consulting fees or travel expenses from Danone; Eroski Foundation, Instituto Danone-Spain, and Abbot Laboratories. Dr. Estruch reported receiving grants from ISCIII and olive oil for the trial from Fundacion Patrimonio Comunal Olivarerolduring the conduct of the study and personal fees from Brewers of Europe, Fundación Cerveza y Salud, Interprofesional del Aceite de Oliva, Instituto Cervantes, Pernaud Richar, Fundación Dieta Mediterránea, Wine and Culinary International Forum; nonfinancial support from Sociedad Española de Nutrición and Fundación Bosch y Gimpera; and grants from Uriach Laboratories outside the submitted work. Dr. Pintó reports serving on the board of and receiving consulting personal fees from Sanofi Aventis, Amgen, and Abbott Laboratories; receiving personal lecture fees from Esteve, Lacer, and Rubio laboratories. Victor Micó reports grants from Fundación Cerveza y Salud.

\section{Acknowledgments}

The authors thank the participants for their enthusiastic collaboration, and the PREDIMED-Plus personnel and investigators, as well as all affiliated primary care centers, for their excellent work. We appreciate the English revision by Elaine M. Lilly, PhD.

Ethics Approval: The Research Ethics Committees of all participating centers approved the study protocol, which was conducted following the standards of the Declaration of Helsinki.

Consent to Participate: All participants provided written informed consent.

\section{Author Contributions}

H.S., M.F., and G.C. designed research; C.L., J.W., M.G., J.S.-S., M.A.M.-G., D.C., L.S.-M., and J.K. conducted research; R.E., J.A.M., X.P., C.V., J.V., J.A.T., 
A.D.-L., and H.L. provided essential reagents; H.S. and G.C. analyzed data; H.S. and G.C. wrote the paper; H.S., M.F., and G.C. had primary responsibility for final content. All authors read and approved the final manuscript.

\section{Data Availability}

The data that support the findings of this study are available from the corresponding author (M.F.) and the author H.S. upon reasonable request.

\section{References}

1. Zhong S, Li L, Shen X, et al. An update on lipid oxidation and inflammation in cardiovascular diseases. Free Radic Biol Med. 2019;144:266-278. doi:10.1016/j.freeradbiomed.2019.03.036

2. Libby P, Kobold S. Inflammation: a common contributor to cancer, aging, and cardiovascular diseases-expanding the concept of cardio-oncology. Cardiovasc Res. 2019;115:824-829. doi:10.1093/cvr/cvz058

3. Ferrucci L, Fabbri E. Inflammageing: chronic inflammation in ageing, cardiovascular disease, and frailty. Nat Rev Cardiol. 2018;15:505-522. doi:10.1038/s41569-018-0064-2

4. Unamuno X, Gómez-Ambrosi J, Rodríguez A, Becerril S, Frühbeck G, Catalán V. Adipokine dysregulation and adipose tissue inflammation in human obesity. Eur J Clin Invest. 2018;48:e12997. doi:10.1111/eci.12997

5. Fedewa MV, Hathaway ED, Ward-Ritacco CL. Effect of exercise training on $\mathrm{C}$ reactive protein: a systematic review and meta-analysis of randomised and non-randomised controlled trials. Br J Sports Med. 2017;51:670676. doi:10.1136/bjsports-2016-095999

6. Palmefors H, DuttaRoy S, Rundqvist B, Börjesson M. The effect of physical activity or exercise on key biomarkers in atherosclerosis-a systematic review. Atherosclerosis. 2014;235:150-161. doi:10.1016/j. atherosclerosis.2014.04.026

7. Parsons TJ, Sartini C, Welsh P, et al. Physical activity, sedentary behavior, and inflammatory and hemostatic markers in men. Med Sci Sports Exerc. 2017;49:459-465. doi:10.1249/MSS.0000000000001113

8. Lee IM, Shiroma EJ, Lobelo F, Puska P, Blair SN, Katzmarzyk PT; Lancet Physical Activity Series Working Group. Effect of physical inactivity on major non-communicable diseases worldwide: an analysis of burden of disease and life expectancy. Lancet. 2012;380:219-229. doi:10.1016/ S0140-6736(12)61031-9

9. Wannamethee SG, Lowe GD, Whincup PH, Rumley A, Walker M, Lennon L. Physical activity and hemostatic and inflammatory variables in elderly men. Circulation. 2002;105:1785-1790. doi:10.1161/ hc1502.107117

10. Zheng G, Qiu P, Xia R, et al. Effect of aerobic exercise on inflammatory markers in healthy middle-aged and older adults: a systematic review and meta-analysis of randomized controlled trials. Front Aging Neurosci. 2019;11:98. doi:10.3389/fnagi.2019.00098

11. Cronin O, Keohane DM, Molloy MG, Shanahan F. The effect of exercise interventions on inflammatory biomarkers in healthy, physically inactive subjects: a systematic review. QJM. 2017;110:629-637. doi:10.1093/ qjmed/hcx091

12. Liberman K, Forti LN, Beyer I, Bautmans I. The effects of exercise on muscle strength, body composition, physical functioning and the inflammatory profile of older adults: a systematic review. Curr Opin Clin Nutr Metab Care. 2017;20:30-53. doi:10.1097/ MCO.0000000000000335

13. Bonaccio M, Pounis G, Cerletti C, Donati MB, Iacoviello L, de Gaetano G; MOLI-SANI Study Investigators. Mediterranean diet, dietary polyphenols and low grade inflammation: results from the MOLI-SANI study. Br J Clin Pharmacol. 2017;83:107-113. doi:10.1111/bcp.12924

14. Smidowicz A, Regula J. Effect of nutritional status and dietary patterns on human serum C-reactive protein and interleukin- 6 concentrations. Adv Nutr. 2015;6:738-747. doi:10.3945/an.115.009415

15. Chan R, Yu B, Leung J, Lee JSW, Woo J. Association of dietary patterns with serum high-sensitivity C-reactive protein level in community-dwelling older adults. Clin Nutr ESPEN. 2019;31(xxxx):38-47. doi:10.1016/j. clnesp.2019.03.004
16. Casas R, Urpi-Sardà M, Sacanella E, et al. Anti-inflammatory effects of the Mediterranean diet in the early and late stages of atheroma plaque development. Mediators Inflamm. 2017;2017:3674390. doi:10.1155/2017/3674390

17. Casas R, Sacanella E, Urpí-Sardà M, et al.; Rosa Ma Lamuela-Raventos. The effects of the Mediterranean diet on biomarkers of vascular wall inflammation and plaque vulnerability in subjects with high risk for cardiovascular disease. A randomized trial. PLoS One. 2014;9:e100084. doi:10.1371/journal.pone. 0100084

18. Richard C, Couture P, Desroches S, Lamarche B. Effect of the Mediterranean diet with and without weight loss on markers of inflammation in men with metabolic syndrome. Obesity (Silver Spring). 2013;21:51-57. doi:10.1002/oby.20239

19. Welty FK, Alfaddagh A, Elajami TK. Targeting inflammation in metabolic syndrome. Transl Res. 2016;167:257-280. doi:10.1016/j.trsl.2015.06.017

20. Bloomfield HE, Koeller E, Greer N, MacDonald R, Kane R, Wilt TJ. Effects on health outcomes of a Mediterranean diet with no restriction on fat intake. Ann Intern Med. 2016;165(7):491. doi:10.7326/m16-0361

21. Tsoupras A, Lordan R, Zabetakis I. Inflammation, not cholesterol, is a cause of chronic disease. Nutrients. 2018;10(5):604. doi:10.3390/ nu10050604

22. Mayr HL, Itsiopoulos C, Tierney AC, et al. Ad libitum Mediterranean diet reduces subcutaneous but not visceral fat in patients with coronary heart disease: a randomised controlled pilot study. Clin Nutr ESPEN. 2019;32:61-69. doi:10.1016/j.clnesp.2019.05.001

23. D'Innocenzo S, Biagi C, Lanari M. Obesity and the Mediterranean diet: a review of evidence of the role and sustainability of the Mediterranean diet. Nutrients. 2019;11(6):1306. doi:10.3390/nu11061306

24. Schröder H, Cárdenas-Fuentes G, Angel Martínez-González M, et al. Effectiveness of the physical activity intervention program in the PREDIMED-Plus study: a randomized controlled trial. Int J Behav Nutr Phys Act. 2018;15(1):1-13. doi:10.1186/s12966-018-0741-x

25. Madssen E, Skaug EA, Wisløff U, Ellingsen Ø, Videm V. Inflammation is strongly associated with cardiorespiratory fitness, sex, BMI, and the metabolic syndrome in a self-reported healthy population: HUNT3 Fitness Study. Mayo Clin Proc. 2019;94:803-810. doi:10.1016/j. mayocp.2018.08.040

26. Kitahara CM, Trabert B, Katki HA, et al. Body mass index, physical activity, and serum markers of inflammation, immunity, and insulin resistance. Cancer Epidemiol Biomarkers Prev. 2014;23:2840-2849. doi:10.1158/1055-9965.EPI-14-0699-T

27. Kantor ED, Lampe JW, Kratz M, White E. Lifestyle factors and inflammation: associations by body mass index. PLoS One. 2013;8:e67833. doi:10.1371/journal.pone.0067833

28. Strohacker K, Wing RR, McCaffery JM. Contributions of body mass index and exercise habits on inflammatory markers: a cohort study of middleaged adults living in the USA. BMJ Open. 2013;3(5):1-8. doi:10.1136/ bmjopen-2013-002623

29. Ko G, Davidson LE, Brennan AM, Lam M, Ross R. Abdominal adiposity, not cardiorespiratory fitness, mediates the exercise-induced change in insulin sensitivity in older adults. PLoS One. 2016;11:e0167734. doi:10.1371/journal.pone. 0167734

30. Park YM, Zhang J, Steck SE, et al. Obesity mediates the association between Mediterranean diet consumption and insulin resistance and inflammation in US adults. J Nutr. 2017;147:563-571. doi:10.3945/jn.116.243543

31. Martínez-González MA, Buil-Cosiales P, Corella D, et al.; PREDIMEDPlus Study Investigators. Cohort profile: design and methods of the PREDIMED-Plus randomized trial. Int J Epidemiol. 2019;48:387-388o. doi:10.1093/ije/dyy225

32. Sayón-Orea C, Razquin C, Bulló M, et al. Effect of a nutritional and behavioral intervention on energy-reduced Mediterranean diet adherence among patients with metabolic syndrome: interim analysis of the PREDIMEDPlus randomized clinical trial. J Am Med Assoc. 2019;322:1486-1499. doi:10.1001/jama.2019.14630

33. Salas-Salvadó J, Díaz-López A, Ruiz-Canela M, et al. Effect of a lifestyle intervention program with energy-restricted Mediterranean diet and exercise on weight loss and cardiovascular risk factors: one-year results of the 
PREDIMED-Plus trial. Diabetes Care. 2019;42(5):777-788. doi:10.2337/ dc18-0836

34. Alberti KG, Eckel RH, Grundy SM, et al.; International Diabetes Federation Task Force on Epidemiology and Prevention; National Heart, Lung, and Blood Institute; American Heart Association; World Heart Federation; International Atherosclerosis Society; International Association for the Study of Obesity. Harmonizing the metabolic syndrome: a joint interim statement of the International Diabetes Federation Task Force on Epidemiology and Prevention; National Heart, Lung, and Blood Institute; American Heart Association; World Heart Federation; International Atherosclerosis Society; and International Association for the Study of Obesity. Circulation. 2009;120:1640-1645. doi:10.1161/CIRCULATIONAHA.109.192644

35. Molina L, Sarmiento M, Peñafiel J, et al. Validation of the REGICOR Short Physical Activity Questionnaire for the adult population. PLoS One. 2017;12:e0168148. doi:10.1371/journal.pone.0168148

36. Ainsworth BE, Haskell WL, Whitt MC, et al. Compendium of physical activities: an update of activity codes and MET intensities. Med Sci Sports Exerc. 2000;32(9 Suppl):S498-S504. doi:10.1097/00005768-200009001-00009

37. Schröder H, Fitó M, Estruch R, et al. A short screener is valid for assessing Mediterranean diet adherence among older Spanish men and women. J Nutr. 2011;141:1140-1145. doi:10.3945/jn.110.135566

38. Baron RM, Kenny DA. The moderator-mediator variable distinction in social psychological research: conceptual, strategic, and statistical considerations. J Pers Soc Psychol. 1986;51:1173-1182. doi:10.1037//0022-3514.51.6.1173

39. Lynch KG, Cary M, Gallop R, Ten Have TR. Causal mediation analyses for randomized trials. Health Serv Outcomes Res Methodol. 2008;8:57_ 76. doi:10.1007/s10742-008-0028-9

40. Whittle R, Mansell G, Jellema P, van der Windt D. Applying causal mediation methods to clinical trial data: what can we learn about why our interventions (don't) work? Eur J Pain. 2017;21:614-622. doi:10.1002/ ejp. 964

41. Lee IM, Sesso HD, Ridker PM, Mouton CP, Stefanick ML, Manson JE. Physical activity and inflammation in a multiethnic cohort of women. Med Sci Sports Exerc. 2012;44:1088-1096. doi:10.1249/ MSS.0b013e318242b11a
42. Item F, Konrad D. Visceral fat and metabolic inflammation: the portal theory revisited. Obes Rev. 2012;13(Suppl 2):30-39. doi:10.1111/j.1467-789X.2012.01035.x

43. Dhaliwal SS, Welborn TA. Measurement error and ethnic comparisons of measures of abdominal obesity. Prev Med (Baltim). 2009;49(2-3):148152. doi:10.1016/j.ypmed.2009.06.023

44. Bosy-Westphal A, Booke CA, Blöcker T, et al. Measurement site for waist circumference affects its accuracy as an index of visceral and abdominal subcutaneous fat in a Caucasian population. J Nutr. 2010;140:954-961. doi:10.3945/jn.109.118737

45. Fuentes GC, Castañer O, Warnberg J, et al. Prospective association of physical activity and inflammatory biomarkers in older adults from the PREDIMED-Plus study with overweight or obesity and metabolic syndrome. Clin Nutr. 2020;39:3092-3098. doi:10.1016/j. clnu.2020.01.015

46. Nilsson A, Bergens O, Kadi F. Physical activity alters inflammation in older adults by different intensity levels. Med Sci Sports Exerc. 2018;50:15021507. doi:10.1249/MSS.0000000000001582

47. Reilly SM, Saltiel AR. Adapting to obesity with adipose tissue inflammation. Nat Rev Endocrinol.2017;13:633-643. doi:10.1038/nrendo.2017.90

48. Bailey MA, Holscher HD. Microbiome-mediated effects of the Mediterranean diet on inflammation. Adv Nutr. 2018;9:193-206. doi:10.1093/advances/nmy013

49. Simioni C, Zauli G, Martelli AM, et al. Oxidative stress: role of physical exercise and antioxidant nutraceuticals in adulthood and aging. Oncotarget. 2018;9(24):17181-17198. doi:10.18632/oncotarget.24729

50. Bird SR, Hawley JA. Update on the effects of physical activity on insulin sensitivity in humans. BMJ Open Sport Exerc Med. 2016;2:e000143. doi:10.1136/bmjsem-2016-000143

51. Camargo A, Delgado-Lista J, Garcia-Rios A, et al. Expression of proinflammatory, proatherogenic genes is reduced by the Mediterranean diet in elderly people. Br J Nutr. 2012;108:500-508. doi:10.1017/ S0007114511005812

52. Llorente-Cortés V, Estruch R, Mena MP, et al. Effect of Mediterranean diet on the expression of pro-atherogenic genes in a population at high cardiovascular risk. Atherosclerosis. 2010;208:442-450. doi:10.1016/j. atherosclerosis.2009.08.004 